\title{
El empleo: factor determinante del desarrollo humano
}

\author{
Mery Ruiz-Guajala1, Kléber Sinchigalo- Guamán²
}

Fecha de recepción: 28 de Mayo 2018

\section{Resumen}

Este artículo surge por la necesidad de analizar la relación del empleo con el Índice de Desarrollo Humano (IDH) en el Ecuador en base a la disponibilidad de datos de fuentes oficiales desde el año 2005 a 2017. Se describe el comportamiento de las variables a lo largo del tiempo y se realiza el análisis econométrico donde se estructuran los modelos Mínimos Cuadrados Ordinarios y ARIMA. Los resultados de este estudio demuestran que el empleo es un factor fundamental para el desarrollo humano y el bienestar social.

Palabras claves: Índice de desarrollo humano, empleo, bienestar social.

\section{Abstract}

This article arises from the need to analyse the employment relationship with the Human Development Index (HDI) in Ecuador in availability of data from official sources since 2005-2017. The behavior of the variables over time is described and the economic analysis is carried out where the Ordinary Least Squares and ARIMA models are structured. The results of this study show that employment is a fundamental factor for human development and social welfare.

Keywords: Human development index, employment, social welfare

\section{Introducción}

Desde los inicios de la década de los 90, han surgido diferentes paradigmas del desarrollo humano y el bienestar social, cuestionando a la teoría económica moderna de la acumulación de capitales, tanto en el sector empresarial como en el ámbito del individuo, donde por su naturaleza el hombre siempre querrá lucrarse. Considerando que la teoría del desarrollo ha dejado rezagado a los indicadores macroeconómicos como el Producto Interno Bruto (PIB) un indicador del desarrollo económico. El aumento de los debates y críticas sobre la debilidad del PIB se fundamenta por numerosos motivos, entre ellos se destacan: no considerar aspectos como la producción, auto subsistencia, así como, los mecanismos de las externalidades por degradación del medioambiente, índice de criminalidad, acceso a servicios de educación y salud, esperanza de vida al nacer, renta y consumo sustentable entre otros (Ibañez, Mujica, \& Castillo, 2017). En ese sentido algunos economistas del desarrollo empezaron a cuantificar estos componentes.

Aquellos componentes son variables que se agrupan en subindicadores, y éstos a su vez, se centran en indicadores que calculan ciertas dimensiones, y con la unificación de los índices de las dimensiones se cuantificaría el desarrollo. De la misma forma, se han incorporado los términos de sustentabilidad y sostenibilidad como sinónimos dentro del desarrollo humano. Esta autonomía de los diferentes componentes de la sustentabilidad desarrolló algunos índices compendiados para medir las dimensiones del desarrollo sustentable ONU (2000). Para este enfoque algunos economistas no han diferenciado los conceptos de desarrollo económico y crecimiento económico, la primera mide el bienestar y nivel de vida de las personas, mientras que la segunda mide el crecimiento porcentual de una economía. En este estudio se enfoca al análisis del primer concepto.

Algunos científicos como Haq (1976), Sen (1999) y Pedrajas (2005), del "desarrollo" han estudiado los diferentes contextos que debe enfrentar el ser humano, desde el momento de su concepción. Ellos estudian la manera en que las personas cambian durante su ciclo de vida. Es desde allí, que surge la necesidad de crear, por decirlo de algún modo, el estudio y análisis formal del desarrollo humano (Diane E, Olds, \& Feldman,
Fecha de aceptación: 29 de Junio 2018

2004). El debate sobre desarrollo humano por parte del economista Sen fue significativo e innovador al indicar, que el proceso de desarrollo es una ampliación de las capacidades humanas como las posibilidades y derechos para acceder a ciertos bienes y servicios que son fundamentales en el desarrollo personal y colectivo, y no como una ampliación del bienestar y de satisfacción económica (Torres \& Allepuz, 2009).

Las desigualdades sociales, son problemas que han causado muchos debates a nivel internacional en diferentes foros, conferencias organizadas por las Naciones Unidas, estas discusiones surgen desde el mismo instante que Amartya Sen dice que las condiciones sociales a través de los tiempos, son desear las igualdades para los sectores periféricos en donde abunda la pobreza en la mayoría de los países, incluyendo los países llamados del primer mundo. La pluralidad social al momento de buscar la igualdad y la libertad es por la realización propia de identificar el valor de las cosas, tangibles o intangibles (Mollina \& Pascual, 2014). De ahí, surge el paradigma de las libertades reales y el desarrollo del ser humano.

El paradigma del desarrollo debe ser visto como un ataque selectivo a las peores formas de pobreza, definirse en términos de reducción continua y eliminación de la desnutrición, la enfermedad, el analfabetismo, el infortunio, el desempleo y las desigualdades (Haq, 1976), que en su esencia filosófica del bienestar social está encaminado a la dignidad humana. El capital humano está basado en modelos de investigación establecidos en el proceso de aprendizaje a través de diferentes factores que no son cuantificables, así como los hábitos de las personas dentro de una sociedad, lo que trasciende de manera significativa, también se puede hacer una valoración a través de la variable proxy; es decir, que se puede medir el empleo y su incidencia en IDH.

El desarrollo humano es la ampliación de las opciones de las personas, acrecentando las funciones y capacidades humanas. Las funciones de ser humano se refieren a las formas valiosas que la persona puede desarrollar o ser, mientras que las capacidades humanas representan varias combinaciones de funcionamientos que pueden llegar alcanzar (Veres, 2006), entonces el concepto de desarrollo humano es mucho más profundo y rico, que algunos índices compuestos o, incluso, en un conjunto de indicadores estadísticos.

En el informe de desarrollo humano de 1990 del programa de la Naciones Unidas para el desarrollo (PNUD) se prepararon diferentes índices compuestos que miden este aspecto, básicamente crear un índice de desarrollo humano como tal. El programa define al IDH como la medición de la calidad de vida del ser humano en el ambiente en que se desenvuelve, y como una forma de calificar el desempeño de un país o región en cuanto a la calidad de vida se refiere o al grado de satisfacción de las necesidades humanas.

El PNUD cada año calcula el IDH, basándose en tres indicadores: esperanza de vida al nacer, nivel de educación y el nivel de ingreso per cápita ajustado. Su valor se ubica entre cero y uno, los países que están cerca a cero menos desarrollo humano tienen, mientras que las naciones con valor del IDH cerca a uno, tienen un desarrollo humano alto como es el caso de Noruega, Austria, Suiza entre otros. El valor del IDH de un país o región muestra una noción, a escala, de su grado de desarrollo y de su clasificación a nivel internacional (Torres \& Allepuz, 2009). Dicha clasificación internacional ha evidenciado desigualdades sociales y económicas.

En los países con bajo valor en el IDH, existe un bajo nivel de empleo, 
que, para algunos analistas económicos, un alto nivel de desempleo es observado como un signo de la debilidad de una economía. La falta de empleo o su mala calidad es tal vez la relación más clara entre vulnerabilidad y pobreza ya que los ingresos económicos derivados del trabajo simboliza la fuente más importante para la sobrevivencia de los hogares (CEPAL, 2000), para muchas familias contar con un empleo estable garantiza la estabilidad económica.

Para el premio nobel de Economía en 2001, Stiglitz (2002), el empleo es una actividad económica que desarrolla el bienestar de los individuos y las estructuras económicas de empresas y de las naciones. Del mismo modo, el premio nobel de economía de 1969, Tinbergen, describe al empleo como una relación gradual de la subutilización de la mano laboral, en los países en vías de desarrollo no se conoce con claridad los niveles de empleo y la subutilización de la mano laboral (Tinbergen, 2013). La escasez del personal con mano de obra calificado y los bajos niveles de los conocimientos tecnológicos contribuirían al aumento del desempleo.

El trabajo y la calidad del empleo son importes en el mundo laboral de hoy, la competitividad laboral y los niveles salariales dependen de las capacitaciones y el nivel académico de los trabajadores, se necesita tener conocimientos básicos, de seguridad industrial, relaciones humanas entre otras. La calidad del empleo se definiría como el conjunto de factores afines del trabajo que intervienen en el bienestar económico, social, psíquico y de salud de la clase obrera (Pérez \& Mora, 2014). Estos factores dependerían básicamente de las condiciones laborales establecidas por las legislaciones de cada uno de los países, y que no se no vulneren los derechos adquiridos por los trabajadores.

A partir de esta teoría surge la necesidad de investigar el empleo y su incidencia en el IDH en el Ecuador desde el año 2005 a 2017, para el empleo se consideraron tres variables: la población económicamente activa (PEA) personas con aptitud y capacidad de trabajar a partir de los 15 años hasta los 65 años de edad de acuerdo a la Constitución Ecuatoriana, tasa de empleo de personas que trabajan en diferentes sectores ya sea público o privado y/o autónomos y dependientes; es decir, el total de la PEA que tienen empleo, y los asalariados totales que son empleos en los que los trabajadores tienen contratos implícitos o explícitos, por los que reciben una remuneración básica que no depende directamente de los ingresos de la unidad para la que trabajan (OIT, 2013).

\section{El empleo y el Índice de Desarrollo Humano en el Ecuador}

En el Ecuador, el IDH según el informe del PNUD (2017) aumentó de 0.739 a 0.743 para el año 2016 ubicándose en el puesto 89 de 188 países y territorios. Desde el 2005 al 2017 el IDH del Ecuador refleja incrementos que se observan en la figura1, por lo que se podría suponer una mejora en la calidad de vida y bienestar social de los ecuatorianos. El reajuste de datos realizado en el 2010 sobre educación, salud e ingreso per cápita por habitante ha promovido el auge económico y social del país.

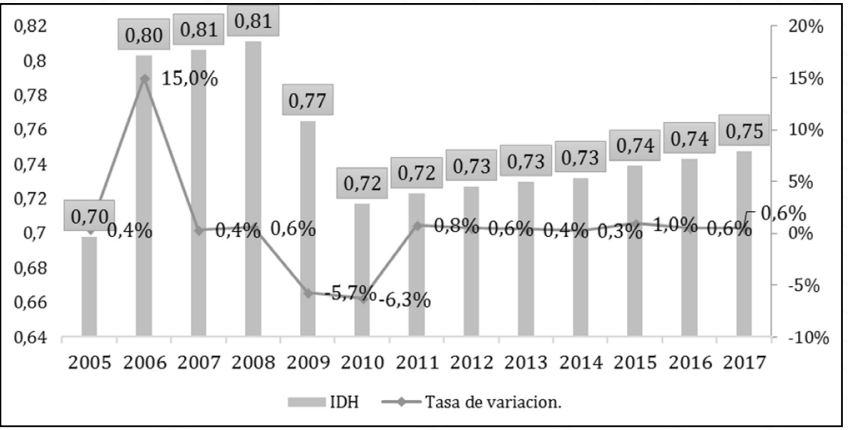

Fuente: Elaboración propia con los datos del PNUD (2018) Figura 1. Índice de Desarrollo Humano del Ecuador y su variación
Ecuador cuenta con buen desempeño en el indicador referente a salud puesto que la esperanza de vida es de 75,87 años y ha aumentado 1,73 años desde el 2005. En el caso de los indicadores de escolaridad, ha variado progresivamente, por ejemplo, ese aumento se puede observar en el porcentaje de alfabetización. De acuerdo con los datos de la Organización de las Naciones Unidas para la Educación, la Ciencia y la Cultura (UNESCO) la meta planteada al 2012 fue del 98\% y se alcanzó 99\% superando la meta. Además, se ha mejorado en la calidad de la educación, con el indicador de la cantidad de estudiantes por docente.

La meta propuesta por la UNESCO fue máximo 40 estudiantes por maestro, a nivel mundial se logró establecer 24 alumnos por docente en el mundo y en el Ecuador el cociente de estudiantes llegó al 18\% en promedio. Con respecto al ingreso per cápita, Ecuador ha experimentado avances significativos, en el 2015 el ingreso se registró en USD 5.851, mientras que en el ingreso Nacional Bruto (INB) per cápita para este año fue de USD 11.270, en este caso es importante recordar, que a partir del año 2010 el PNUD utiliza como un nuevo indicador INB en remplazo de Producto Interno Bruto (PIB) per cápita. El INB per cápita en la paridad del poder adquisitivo (PPA) es expresado en dólares internacionales que tiene el mismo PPA con los dólares de los Estados Unidos de América. Ecuador es la economía número 61 en el ranking de 196 países del mundo, además es la octava más importante de Latinoamérica (BCE, 2016).

En cuanto al empleo según el Instituto Nacional de Estadística y Censos (INEC) 2018 existen 11,5 millones de personas que están en edad de trabajar; esa cifra corresponde al 69,6\% de la población total. También se indica que 7,9 millones de ecuatorianos pertenecen a la Población Económicamente Activa (PEA); es decir, que el 95,4 \% de la PEA está trabajando y el desempleo es 4,60 \%. Para algunos analistas económicos, la tasa de desempleo es un indicador para conocer la debilidad o la fortaleza de la economía de un país o región.

Desde el 2005 al 2017 la PEA del Ecuador presenta una disminución promedio del 1,07\% (Figura 2), lo que significa que ha disminuido la población en capacidad de trabajar. Además, el año con menor tasa de empleo fue 2005, y en el año 2014 se observó 96,2\% de empleo.

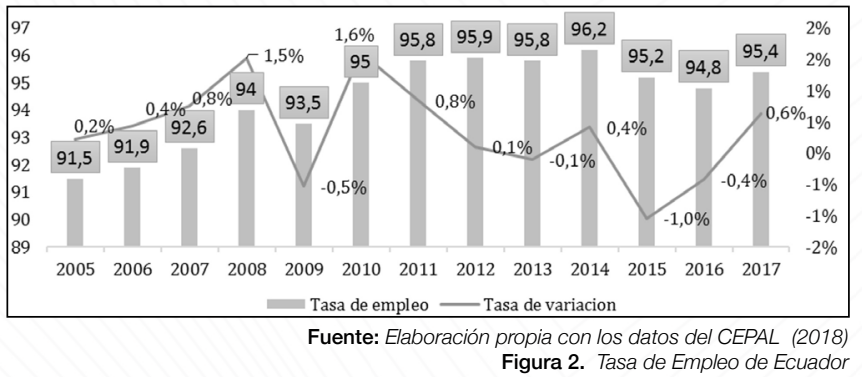

Más allá de las cifras económicas, el país está en pleno desarrollo económico y social, lo que constituye que el país tiene el desafío de brindar una vida digna para su población. Por tanto, se pretende conocer el comportamiento de las variables de estudio y de acuerdo al problema planteado en la presente investigación, se consideran las siguientes hipótesis.

HO: "El empleo no incide en el índice de desarrollo humano en el Ecuador"; H1: "El empleo incide en el índice de desarrollo humano en el Ecuador".

\section{Metodología}

La búsqueda de la información se realizó de fuentes oficiales del PNUD de donde se obtuvieron datos del IDH, el dato referente a la tasa del empleo se obtuvo del portal de información "Ecuador en Cifras" publicado por el INEC y CEPAL, finalmente la fuente más importante de este trabajo investigativo fue el Banco Central del Ecuador (BCE).

Para la estructuración de los modelos econométricos se utilizó el software Gretl de libre distribución y utilizado por los académicos de las ciencias de la Matemática, Estadística y Econometría. El modelo de Mínimos Cuadrados Ordinarios (MCO) es un modelo lineal común y matemáticamente fácil de describir. En este modelo se encontró que las variables explicativas no son estacionarias por lo que se tuvo que aplicar pruebas 
estadísticas de serie temporal como la prueba de Dickey-Fuller (Gujarati \& Porter, 2009, págs. 754-755) y los test estadísticos de Dickey-Fuller Aumentado para conocer las debilidades que se podrían presentar en el proceso de predicciones (Maddala, 1992, pág. 532).

Además, se hizo la prueba de cointegración de Engle-Granger, Johansen que se refiere a la relación entre variables no estacionarias con raíz unitaria; es decir, cuando dos series están cointegradas a pesar de que una de las variables pueda ser estacionaria, existe un equilibrio a largo plazo (Granados, 2013). Luego de las pruebas de serie temporal, se procedió a la estructuración de los modelos. El modelo MCO es integrado, y econométricamente se formuló de la siguiente forma:

$$
\begin{array}{ll}
\text { Donde: } & \mathrm{X}_{1}=\text { Población económicamente activa. (PEA) } \\
\beta_{0}=\text { constante } & \text { de orden cero } \\
\beta_{1}, \beta_{2}=\text { Coeficientes } & d \mathrm{X}_{2}=\text { Tasa del empleo de orden uno } \\
d \mathrm{Y}_{1}=\text { Índice de Desarrollo Humano (IDH), de } & \mu_{1}=\text { Error de perturbación } \\
\text { orden uno } &
\end{array}
$$$$
d \mathrm{Y}=\beta_{0}+\beta_{1} \mathrm{X}_{1}+\beta_{2} d \mathrm{X}_{2}+\mu_{1}
$$

En este modelo también se estructuró las pruebas estadísticas de Test de RESET Ramsey para determinar si el modelo está correctamente especificado; es decir, que se pretende comprobar sí, en el modelo se omitieron variables de relevancia o si se identificaron variables irrelevantes. Además, se realizó el contraste de Autocorrelación de Breusch-Godfrey para conocer si el comportamiento de una variable puede explicarse por sí misma. Otra de las pruebas aplicadas fue la de colinealidad, donde se puede conocer si las variables regresoras se encuentran correlacionadas.

Se realizó la prueba de heterocedasticidad de White para que la varianza no se mantenga constante en todas las observaciones de la muestra. Por la presencia en los datos de no estacionalidad se estructuró el modelo Autorregresico Integrado de promedios Móviles (ARIMA) y econométricamente se formuló de la siguiente forma:

$$
\begin{array}{ll}
\qquad d Y_{1}=\beta_{0}+\beta_{1} Y_{1(t-1)}+\beta_{2} \mu_{t-1}+\beta_{3} d_{-} X_{1}+\beta_{4} d_{-} X_{2}+\beta_{5} d_{-} X_{3}+\mu \\
\text { Donde: } & \\
d Y_{1}=\text { Primera diferencia del IDH } & d_{-} X_{1}=\text { PEA integrada de orden Uno } \\
\beta_{0}=\text { constante } & d_{-} X_{2}=\text { Tasa de empleo integrada de orden Uno } \\
\beta_{1}, \beta_{2}, \beta_{3}, \beta_{4}, \beta_{5}=\text { Coeficientes } & d_{-} X_{3}=\text { Asalariados de integrada de orden Uno } \\
Y_{1(t-1)=I D H ~(2006-1)} & \mu=\text { Error de perturbación } \\
\mu_{t-1}=\text { Error de perturbación }(2006-1) &
\end{array}
$$

A igual que el modelo anterior, se realizaron las pruebas estadísticas. Los dos modelos son significativos, entonces se aplicó el criterio de elección de modelos. Cuando por diversos motivos en un análisis econométrico se tiene que elaborar algunos modelos, el investigador enfrenta la disyuntiva de la elección de un modelo más preciso y adecuado. Para la elección correcta se debe tener en cuenta los objetivos de análisis, la teoría económica utilizada y los resultados estadísticos, además depende de los modelos uniecuacionales y multiecuacionales (Solera, 2000) y los criterios de elección de modelo de Akaike, Schwarzy Hannan Caballero, (2011). El criterio más adecuado es cuando su valor estadístico sea más bajo.

\section{Resultados}

Los resultados de esta investigación se muestran en dos formas: la primera el análisis descriptivo de las variables de estudio y la segunda el análisis econométrico.

El empleo es una variable que constituye un medio de subsistencia que permiten a las personas salir de la pobreza e incrementar su nivel de vida. Las variaciones del empleo a lo largo del tiempo han sido un factor determinante para alcanzar el desarrollo social y económico del país.

Tabla 1. Media, desviación típica y tasa de variación de las variables estudiadas

\begin{tabular}{|cccc|}
\hline Variables & Media & Desviación Típica & $\begin{array}{c}\text { Tasa de variación } \\
\text { promedio }\end{array}$ \\
\hline IDH & 0,749 & 0,036 & 0.57 \\
\hline PEA & 0,687 & 0,009 & $-0,09$ \\
\hline Tasa de empleo & 0,940 & 0,012 & 0,27 \\
\hline Asalariados & 0,491 & 0,047 & $-1,70$ \\
\hline
\end{tabular}

Fuente: Elaboración propia con los datos de PNUD, CEPAL, Banco Mundial, INEC
En esa perspectiva se conocerá el comportamiento de las variables que componen el empleo. En cuanto al IDH en el Ecuador durante el periodo 20052017, ha experimentado una variación promedio de 0,57\%, aunque esto no es significativo con respecto a otros países de la región. El país se ubicó dentro del IDH alto, esto probablemente por las políticas sociales implementadas por el gobierno, que han permitido reducir las brechas de desigualdad. Desde 2005 hasta 2017 en IDH la media es de 0,749 puntos, lo que indica que el país está en el proceso de desarrollo social medio con respecto a Noruega que es uno de los países más desarrollados y equitativos del mundo. Además la dispersión con los datos del promedio es 0,036.

Con respecto a la PEA, el promedio durante este periodo fue del $68,7 \%$; es decir, que la población en capacidad de trabajo es representativa, lo que indica un aumento en la tasa del empleo (tabla 1). En cuanto a los asalariados totales en este periodo de estudio han disminuido en un promedio de $1,70 \%$, es decir, que existe mayor población que percibe sueldos superiores al salario básico unificado.

En el modelo de MCO se observa que las variables regresoras son estadísticamente significativas, excepto la variable salariados totales $(X 3)$ por lo que se procedió a la omisión de esta , y el modelo subajustado de MCO integrado es el correcto; así como lo demuestra el estadístico Fisher-Snedecor con un valor de probabilidad de 0,00104, con lo que se entiende que el modelo en su conjunto es estadísticamente significativo, además, el coeficiente de determinación ajustado indica un valor de 0,840857 , comprobando que las variables explicativas del PEA (X1) y la tasa de empleo de orden uno (d_X2) explican en un 84,09\% a la variable explicada de IDH orden uno (d_Y1).

Tabla 2. MCO, variable IDH del orden uno en el periodo 2005-2017

\begin{tabular}{cccccc}
\hline & Coeficiente & $\begin{array}{c}\text { Desv. } \\
\text { Típica }\end{array}$ & Estadístico t & valor p & \\
\hline const & $-2,71331$ & 0,351699 & $-7,7149$ & $<0,0001$ & $* * *$ \\
X1 & 3,9599 & 0,512316 & 7,7294 & $<0,0001$ & $* * *$ \\
d_X2 & $-0,998699$ & 0,513186 & $-1,9461$ & 0,0835 & $*$ \\
\hline $\begin{array}{l}\text { R-cuadrado corregido } \\
\text { Valor p (de F) }\end{array}$ & 0,840857 & & & & \\
\hline & 0,000104 & \multicolumn{5}{c}{ Fuente: Elaboración Propia }
\end{tabular}

La variable estimada y observada contra la variable exógena del PEA y tasa de empleo del primer orden es estadísticamente significativa; es decir, el IDH estimado con respecto a las variables explicadas muestra que la hipótesis es nula, por tanto hay una incidencia con las variables explicativas del empleo.

En cuanto al modelo ARIMA es necesario conocer el correlograma para analizar los valores de la Función de Autocorrelación (FAC) y de la Función de Autocorrelación Parcial (FACP) con el objetivo de identificar el orden del componente Autorregresivo (AR) y de Medias Móviles (MA) como se puede apreciar en el gráfico siguiente:

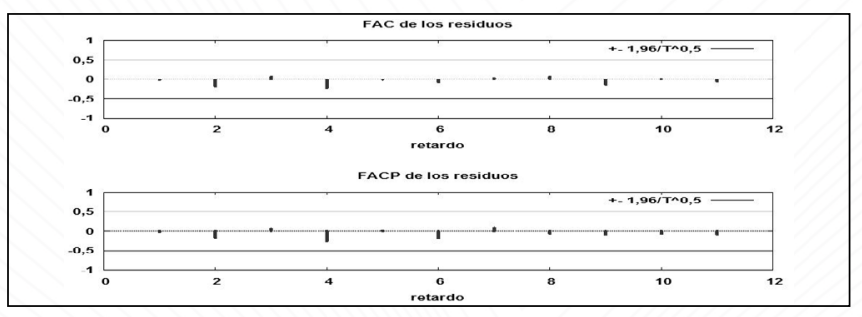

Fuente: Elaboración Propia desde el software Gretl Figura 3. Análisis de correlograma del modelo integrado

No se visualiza ningún valor de FAC significativo puesto que ninguno de estos sobresale a los límites de confianza (figura 3), por lo tanto, se puede argumentar que la FAC sigue una tendencia sinusoidal. Así mismo, ningún valor de FACP sobresale de los límites de confianza; es decir, que la FACP también sigue una tendencia sinusoidal.

Se determina que el orden del Modelo Autorregresivo Integrado de Promedios Móviles (ARIMA) es (1, 1, 1), es decir, que el componente autorregresivo se estima en función de su primer rezago, al igual que el componente de medias móviles, estimado en función del rezago del orden uno de los residuos de la regresión. 
Tabla 3. Modelo ARIMA usando las variables 2006-2017

\begin{tabular}{lccccl}
\hline & Coeficiente & Desv. Típica & $\mathbf{z}$ & valor $\mathbf{p}$ & \\
\hline const & 0,0147922 & 0,0179179 & 0,8256 & 0,4091 & \\
phi_1 & 0,439546 & 0,29434 & 1,4933 & 0,1354 & \\
theta_1 & 1 & 0,257537 & 3,8829 & 0,0001 & $* * *$ \\
X1 & 2,34578 & 0,569256 & 4,1208 & $<0,0001$ & $* * *$ \\
X2 & $-0,979485$ & 0,532569 & $-1,8392$ & 0,0659 & $*$ \\
X3 & 0,329833 & 0,18062 & 1,8261 & 0,0678 & $*$ \\
\hline
\end{tabular}

Fuente: Elaboración Propia desde el software Gretl

En el modelo ARIMA las variables explicativas de la PEA (X1), Tasa de empleo (X2) y asalariados (X3) son estadísticamente significativas; es decir, que las variables explicativas inciden en la variable endógena, además la media móvil (theta_1) es estadísticamente significativa con un valor de probabilidad de 0,0001; es decir, que los rezagos de los residuos inciden con el comportamiento de la variable explicada. Mientras que el componente autoregresivo (phi_1) no es estadísticamente significativo; es decir, que los rezagos de los residuos no inciden con el comportamiento de la variable IDH. Por lo tanto el modelo es de Medias Móviles.

Para poder establecer con mayor exactitud la elección de modelos econométricos se aplicó los criterios de información bayesiano o criterio de Schwarz, criterio de información Akaike y criterio de información Hannan-Quinn para el modelo Integrado y modelo Medias Móviles.

Tabla 4. Criterios de elección de modelos

\begin{tabular}{ccc}
\hline Criterios & Modelo MOC Integrado & $\begin{array}{c}\text { Modelo ARIMA } \\
\text { (Medias Móviles) }\end{array}$ \\
\hline Schwarz & $-62,83434$ & $-40,81510$ \\
Akaike & $-64,28906$ & $-44,20945$ \\
Hannan-Quinn & $-64,82765$ & $-45,46616$ \\
\hline & Fuente: Elaboración Propia desde el software Gretl
\end{tabular}

Los criterios ya mencionados son introducidos para penalizar el exceso de parámetros ajustados, por lo tanto estos criterios son muestrales, de acuerdo a lo mencionado, el criterio de Hannan-Quinn tiene una analogía al criterio de información bayesiano (CIB) que indica que es la más precisa para el modelo integrado con un valor de -64,82765; es decir, que el orden de autorrelación es mínima por lo tanto, mientras para el modelo Medias Móviles el criterio Hannan-Quinn tiene un valor estadístico de -45,46616, la elección del modelo de acuerdo a estos criterios se inclina al modelo de (MCO) integrado; por tanto, para la aplicación del mismo se consideró la primera diferencia de los valores naturales.

\section{Conclusiones}

Sin duda el desarrollo humano, y sobre todo la calidad de vida siempre dependerá de los ingresos económicos y para ellos es indispensable contar con una fuente de trabajo. El empleo como parte fundamental para el desarrollo económico y social de un país indica el fortalecimiento de una economía. El dinamismo económico siempre estará enfocado a la productividad que pueda desarrollar el ciudadano, teniendo en cuenta su capacidad física y/o mental, lo que ayuda para el desarrollo de sí mismo y de su familia. En ese sentido se llega a concluir que el empleo incide de manera representativa en el índice de desarrollo humano.

En cuanto a la tasa de empleo para el año 2005, el 91,5\% del total de la PEA tenía trabajo, mientras que para el año 2017 se registró 94,5\%; es decir, el comportamiento de la tasa de empleo presentó un incremento de $0,27 \%$ (tabla 1). Así mismo con respecto a los asalariados totales se observó una disminución del 1,70\% de los trabajadores con sueldo básico. Por lo que se concluye que la disminución de la PEA fue un indicador que influyó en el aumento del salario básico unificado, es decir, que existe más empleados que tienen un ingreso económico por encima de la base establecido por la ley; esto es positivo porque existe mayor dinamismo económico.

Ecuador para el año 2016 se ubicó en el puesto 89 del ranking IDH, una ubicación que representa estar en un nivel sobresaliente. Por lo tanto, se concluye que el comportamiento del IDH en el Ecuador durante 12 años presenta un incremento promedio de 0,57\% (tabla1), lo que indica que existe una mejor calidad de vida, mejor redistribución de los recursos por parte del Estado Ecuatoriano.

En esta investigación se aplican dos modelos econométricos, el MCO y ARIMA, y debido a que las variables no son estacionarias, se tomó en cuenta los criterios de selección de modelos. Para el modelo MCO con las variables de primer orden se concluye que las dos primeras variables explicativas PEA y tasa de empleo son estadísticamente significativas; es decir, existe una relación directa con la variable explicada del IDH; y la variable asalariados no es significativa.

Para el caso de modelo ARIMA el IDH con las variables explicativas son todas estadísticamente significativas, es decir, que el modelo es correcto. De acuerdo a los resultados, el modelo es de Medias Móviles (tabla 3), porque el phi_1 o componente autoregresivo no es estadísticamente significativo, se concluye que el modelo es de Medias Móviles. Los dos modelos anteriores son correctos y estadísticamente significativos, y con cualquiera de ellos se puede realizar proyecciones, sin embargo, cuando se presenta esta disyuntiva, se debe considerar la esencia del estudio, para la determinación del modelo más preciso. En este caso el modelo MCO integrado es el más adecuado, según el criterio de Hannan-Quinn con valor de $-64,82765$. Se concluye que la PEA y la tasa de empleo inciden significativamente con el Índice de Desarrollo Humano en el Ecuador y bajo estos resultados se puede realizar proyecciones con mayor exactitud.

\section{Referencias}

BCE. (2016). Banco entral del Ecuador. Obtenido de Banco central del Ecuador: https://www.bce.fin.ec/index.php/informacion-estadistica-1 CEPAL. (2000). Panorama social de América Latina 1999-2000. Chile: Comisión Económica para América Latina y el Caribe. Diane E, p., Olds, S. W., \& Feldman, R. D. (2004). Desarrollo Humano. México D.F: TheMGraw-Hill.

Granados, R. M. (Marzo de 2013). Test de causalidad. Obtenido de Test de causalidad: http://Www.ugr.es/ montero/matematicas/causalidad.pdf Gujarati, D., \& Porter, D. (2009). Econometria (Quinta edición ed.). (M.

Toledo, J. Chacón, E. Zúñiga, Edits., \& P. Villarreal, Trad.) México FD: McGraw Hill/Irwin, Inc.

Haq, M. u. (1976). The Poverty Curtain. New York: Columbian University Press Ibañez, N . Mujica, M. \& Castillo, R. (2017). Componentes Del Desarrollo Humano Sustentable. Revista Cientifica Electrónica de Ciencias Gerenciales(36), $63 / 77$.

INEC. (21 de 05 de 2018). Obtenido de Instituto Nacional de Estadística y Censo: $h$ ttp://www.ecuadorencifras.gob.ec/vdatos/

Maddala, G. (1992). Intruduction to econometrics. (second ed.). New York: MacMillan Publishing Company.

Mollina, R., \& Pascual, J. (2014). El Índiice De Desarrollo Humano Como indicador Social. Revista Crítica de Ciencias Sociales y Jurídicas(44), 1-18.

OIT. (2013). La Revista Internacional del Trabajo y la OIT. Fragmentos de su Historia. Revista Internacional del Trabajo, 132(Extraordinario), 1-120.

Pedrajas, M. (2005). El Desarrollo Humano en la Economía Ética de Amartya Sen (Tesis Doctoral). Valencia: Universitat de València Servei de publicacions.

Pérez, L., \& Mora, J. (2014). La calidad del empleo en la población afrodescendiente colombiana: una aproximación desde la ubicación geográfica de las comuna. Revista de Economía del Rosario, 17(2), 117-149.

PNUD. (2017). Informe sobre Desarrollo sobre el Desarrollo Humano 2016. Nueva York, NY 10017, Estados Unidos: Lowe-Martin Group. Obtenido de http://hdr.undp.org/sites/default/files/HDR2016_SP_Overview_Web.pdt

Sen, A. (1999). Desarrollo y Libertad. Barcelona: Planeta S.A.

Solera, Á. (2000). Criterio para seleeción de Modelos Econometricos. Banco Central de Costa Rica. Departamento de Investigacion Economica., 1-17. Obtenido de $h$ ttp://www.bccr.fi.cr/ndie/NT-07-2000.PDF

Stiglitz, J. (2002). Empleo, justicia social y bienestar de la sociedad. Revista Internacional del Trabajo, 121(1-2), 9-31.

Tinbergen, J. (2013). Política comercial y crecimiento del empleo. Revista Internacional del Trabajo, 132(extraordinario), 491-497.

Torres, T., \& Allepuz, R. (2009). El desarrollo humano: perfiles y perspectivas futuras. Estudios de Economía Aplicada, 27(2), 545-562.

Veres, E. (2006). Diseño de un índice para la medicion del desarrollo humano. Estudios de Economía Aplicada, 24(3), 1013-1042. 